\title{
Erratum to "Effects of different inspired oxygen fractions on sildenafil-induced pulmonary anti-hypertensive effects in a sheep model of acute pulmonary embolism"
} [Life Sci. 127 (2015) 16-21]

\author{
Diana Rocío Becerra Velásquez ${ }^{\mathrm{a}}$, Francisco José Teixeira-Neto ${ }^{\mathrm{a}, \mathrm{b}}$, Angie Paola Lagos-Carvajal ${ }^{\mathrm{b}}$, \\ Miriely Steim-Diniz ${ }^{\mathrm{b}}$, Nathalia Celeita Rodríguez ${ }^{\mathrm{a}}$, Carlos Alan Dias-Junior ${ }^{\mathrm{c}, *}$ \\ a Departamento de Cirurgia e Anestesiologia Veterinária, Faculdade de Medicina Veterinária e Zootecnia, Universidade Estadual Paulista (UNESP), Botucatu, São Paulo, Brazil \\ b Departamento de Anestesiologia, Faculdade de Medicina, Universidade Estadual Paulista (UNESP), Botucatu, São Paulo, Brazil \\ c Departamento de Farmacologia, Instituto de Biociências de Botucatu, Universidade Estadual Paulista (UNESP), Botucatu, São Paulo, Brazil
}

The authors regret to inform that some inaccuracies were published in the above article.

On page 26, under Abstract, second paragraph, first sentence: "Forty" should read "Thirty-two" and " 4 " should read " 8 " The correct sentence appears below.

"Thirty-two anesthetized, mechanically ventilated sheep (34.9 \pm $5.4 \mathrm{~kg}$ ) were randomly distributed into four groups ( $=8$ per group):"

On page 26, under Abstract, third paragraph, first sentence: "Emb + Sil ${ }_{90}$ " should read "Emb + Sild 90 " and "Emb + Sil 21 " should read "Emb + Sild $_{21}$." The correct sentence appears below.

Compared with values recorded 30 min after induction of APE $\left(E_{30}\right)$, sildenafil induced greater decreases in MPAP in the Emb + Sild 90 group than in the Emb + Sild $_{21}$ group ( $23 \%$ and $14 \%$ lower than $\mathrm{E}_{30}$, respectively). Hypotension (mean arterial pressure $<60 \mathrm{~mm} \mathrm{Hg}$ ) was precipitated by sildenafil due to systemic vasodilation in the Emb + Sild $_{21}$ group.

On page 26, under Material and methods, second paragraph, first sentence: "Forty" should read "Thirty-two". The correct sentence appears below.

"Thirty-two rams of the Santa Inês breed, weighing $34.9 \pm 5.4 \mathrm{~kg}$ (mean $\pm \mathrm{SD}$ ) were grouped in pens ( $3 \mathrm{~m}^{2}$ per animal) with free access to food and water for at least 10 days before the experiments."

On page 26, under Material and methods, third paragraph, first sentence: "with" should read "without". The correct sentence appears below.

Animals were kept without food and water for 24 and $12 \mathrm{~h}$ before the experiment, respectively.
On page 28, Fig. 1: "Sham" should read "Sham 90 ", "Emb" should read "Emb 90 ", Emb + Sild 100 should read Emb + Sild 90 , and "Emb + Sild 21" should read "Emb + Sild $_{21}$."

On page 30, Fig. 2: "Sham" should read "Sham 90 ", "Emb" should read "Emb 90 ", Emb + Sild 100 should read Emb + Sild ${ }_{90}$, and "Emb + Sild 21" should read "Emb + Sild $_{21}$."

On page 29, under Results, fourth paragraph: "Fig. 1" should read "Fig. 2". The correct sentence appears below.

The MVV was increased from baseline after embolization in all groups to maintain $\mathrm{pH}$ and $\mathrm{PaCO}_{2}$ within physiological limits (35-45 $\mathrm{mm} \mathrm{Hg}$ and 7.35 to 7.45, respectively) (Fig. 2 and Table 1).

On page 29, under Results, fifth paragraph, last sentence: "(from $\mathrm{S}_{15}$ to $W_{30}$ )"should read "(from $E_{30}$ to $W_{30}$ )", and " $S_{15}$ time point" should read " $E_{30}$ time point". The correct sentence appears below.

The Emb $\mathrm{Emo}_{90}$ and Emb + Sild ${ }_{90}$ groups presented lower $\mathrm{PvO}_{2}$ than that of the Sham ${ }_{90}$ group after embolization (from $\mathbf{E}_{\mathbf{3 0}}$ to $W_{30}$ ), except for the $\mathbf{E}_{\mathbf{3 0}}$ time point in the Emb + Sid $_{90}$ group.

On page 29, under Results, last paragraph, last sentence: "but significantly higher values were recorded in the Sham 90 group" should read "but values in these groups were significantly higher than those recorded in the Sham 90 group." The correct sentence appears below.

The Qs/Qt in the Emb ${ }_{90}$ group did not differ from the Qs/Qt in the $\mathrm{Emb}+\mathrm{Sild}_{21}$ group after embolization but values in these groups were significantly higher than those recorded in the Sham 90 group.

\footnotetext{
DOI of original article: http://dx.doi.org/10.1016/j.lfs.2015.02.005.

* Corresponding author at: Departamento de Farmacologia, Instituto de Biociências de Botucatu, Universidade Estadual Paulista (UNESP), Distrito de Rubião Junior, S/N, 18.618-970 Botucatu, São Paulo, Brazil. Tel.: +55 1438800214.

E-mail address: carlosjunior@ibb.unesp.br (C.A. Dias-Junior)
} 